\title{
Evaluation of oxidative stress in seminal plasma of adolescents with varicocele
}

\author{
Valéria Barradas', Mariana Pereira Antoniassi D1, Paula Intasqui', Marcilio Nichi³, Ricardo Pimenta Bertolla1,2 and \\ Deborah Montagnini Spaine ${ }^{1}$
}

1Department of Surgery, Division of Urology, Human Reproduction Section, Universidade Federal de São Paulo, São Paulo, Brazil 2Hospital São Paulo, São Paulo, Brazil

3Department of Animal Reproduction, School of Veterinary Medicine and Animal Sciences, University of Sao Paulo, São Paulo, Brazil

Correspondence should be addressed to M P Antoniassi: mariana_antoniassi@hotmail.com

\begin{abstract}
Varicocele, defined by a dilation of efferent testicular veins, is the most commonly identifiable, surgically correctable lesion associated with male-factor infertility, starts at puberty and causes a progressive decline in fertility potential. The pathophysiology of infertility caused by this disease is still poorly understood, but it is suggested that the main mechanism is oxidative stress. Therefore, the aim of this study was to verify if the varicocele is associated with changes in enzymatic antioxidant mechanisms and seminal plasma lipid peroxidation levels in adolescents. We recruited 90 adolescents that were divided into control (C; $n=27$ ); varicocele and normal semen (VNS; $n=46)$; varicocele and altered semen (VAS; $n=17)$. Seminal and serum levels of lipid peroxidation were quantified by thiobarbituric acid reactive substances (TBARS). Seminal plasma antioxidant profile was evaluated by the activities of catalase (CAT), glutathione peroxidase (GPX) and superoxide dismutase (SOD). The VAS group had increased lipid peroxidation levels when compared to the other groups. The levels of serum lipid peroxidation and activities of the enzymes SOD and GPx did not differ between groups. CAT was undetectable by the method used. In conclusion, in adolescents with varicocele and altered semen analysis, there is an increase in seminal lipid peroxidation levels compared to adolescents with varicocele and without seminal change and adolescents without evident varicocele. However, the observed oxidative stress is not caused by a decrease in superoxide dismutase and glutathione peroxidase activities, which did not differ between adolescents with and without evident varicocele.
\end{abstract}

\section{Lay Summary}

Varicocele, defined by a dilation of efferent testicular veins, is the most commonly identifiable, surgically correctable lesion associated with male-factor infertility, starts at puberty and causes a progressive decline in fertile potential. There is still much that is not understood regarding how exactly it affects semen quality, but most studies agree that oxidative stress, which is defined as excessive amounts of free radicals in relation to antioxidant defense, is an important mechanism. In this study, we aimed to verify if the varicocele is associated with changes in antioxidant defense and semen oxidation in 90 adolescents with and without varicocele. In adolescents with varicocele and abnormal semen, there is an increase in semen oxidation compared to controls or to the group with varicocele and normal semen quality. Our results can help to understand how varicocele leads to infertility in adolescents, identifying changes in oxidative activity in semen, since the onset of varicocele and before damage to sperm production can be detected.

Key Words: $\quad$ varicocele $\quad$ oxidative stress $\quad$ antioxidants $\quad$ adolescent $\quad$ seminal analysis

https://raf.bioscientifica.com

https://doi.org/10.1530/RAF-20-0048 (c) 2021 The authors Published by Bioscientifica Ltd

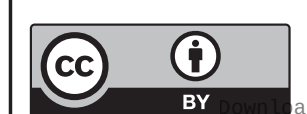

This work is licensed under a Creative Commons Attribution 4.0 International License. 


\section{Introduction}

Infertility, defined as the couple's failure to conceive after 12 months of regular and unprotected sexual intercourse, affects about $15 \%$ of couples in reproductive age and the male factor is associated in up to $50 \%$ of cases (Brugh \& Lipshultz 2004). Varicocele is the main treatable cause of male infertility and is characterized by an abnormal dilation of the pampiniform plexus veins, accompanied by a reversal of blood flow and by venous stasis (Brugh et al. 2003). Its prevalence increases gradually until the age of 15 , when it reaches the same prevalence observed in adults: approximately $15 \%$ of the male population (Gorelick \& Goldstein 1993, Witt \& Lipshultz 1993). Moreover, its prevalence can reach about $35-40 \%$ in primary infertility and $80 \%$ in secondary infertility, suggesting a progressive impairment in the male fertile potential caused by varicocele (Clavijo et al. 2017).

The mechanism of varicocele associated with infertility has not yet been fully understood. However, in order to explain the impaired spermatogenesis commonly observed in these patients, some complementary and interconnected hypotheses have been postulated: (i) an increase in scrotal and testicular temperature; (ii) presence of testicular hypoxia; (iii) presence of reflux in kidney and adrenal metabolites; (iv) decreased perfusion of the affected testicle caused by increased venous pressure; and (v) hormonal dysfunction (Jensen et al. 2017).

The venous stasis caused by varicocele decreases blood flow in the testis, impairing the arterial blood cooling system, thus leading to testicular hyperthermia. Therefore, the metabolism of germ cells is accelerated during spermatogenesis, which, in turn, is not accompanied by an increase in testicular blood supply. Thus, the absence of adequate oxygenation induces a chronic hypoxia state, which causes a direct damage to germ cells as well as activates reactive oxygen species (ROS)-generating metabolic pathways (Hassanin et al. 2018).

An excess in ROS levels is harmful to Leydig, Sertoli and spermatogenic cells, and has a negative impact on several functions of mature sperm (Cho et al. 2016). Furthermore, ROS are able to destabilize the double bonds of polyunsaturated fatty acids (PUFAs), the main component of sperm and mitochondrial membranes, in a chain reaction known as lipid peroxidation. Once established, this process becomes autocatalytic, leading to the formation of several highly toxic metabolites, mainly malondialdehyde (MDA), which has been widely used as a marker of lipid peroxidation (Benedetti et al. 2012, Tsikas 2017). Lipid peroxidation is responsible for serious alterations in sperm function, such as mitochondrial dysfunction and reduced sperm motility and ability to undergo acrosome reaction, in addition to alterations to the sperm morphology (Aitken 2020).

Semen antioxidant defense systems, present in both sperm and seminal plasma, can counteract ROS excessive levels. Under physiological conditions, there is a balance between ROS production and the antioxidant protection. However, when an imbalance occurs in favor of the oxidant, due to a reduction in the antioxidant capacity and/or to increased ROS production, oxidative stress occurs (Alahmar 2019). Sperm are especially susceptible to oxidative stress due to their low cytoplasmic content, insufficient for antioxidant protection, and the high concentration of PUFAs in their plasma membrane, which makes them highly dependent on the antioxidant machinery in the seminal plasma (Aitken et al. 2012). Therefore, the seminal plasma has an important role in supporting sperm function, protecting the male gametes from ROS damaging effects (Szczykutowicz et al. 2019). The antioxidant activity of superoxide dismutase (SOD), catalase (CAT) and glutathione reductase (GPx) protects the sperm membrane against lipid peroxidation in an attempt to preserve its integrity. Thus, the seminal activity of these enzymes associated with MDA levels has been described as effective indicators of oxidative stress (Agarwal \& Majzoub 2017).

Previous studies have shown that young men with varicocele have high ROS levels and sperm lipid peroxidation levels (Romeo et al. 2003; Türkyilmaz et al. 2004). However, it remains to be elucidated how oxidative stress occurs in the seminal plasma of adolescents with varicocele and affects sperm function. Thus, the objective of our study was to determine whether, in adolescents, varicocele is associated with alterations in the enzymatic antioxidant mechanisms and lipid peroxidation levels in the seminal plasma.

\section{Methods}

\section{Study design}

A cross-sectional study was conducted with 128 adolescents, recruited from the National Service of Industrial Learning/ SENAI 'Conde José Vicente de Azevedo' in Sao Paulo, Brazil. The adolescents and their legal guardians were invited to a lecture, and those who agreed to proceed in the study signed informed consent (their own and their legal guardian's). Institutional Review Board approval was obtained from the Sao Paulo Federal University Ethics Committee.

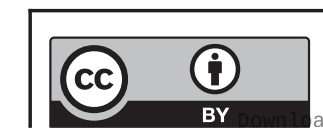


Medical interview and genital physical examination were performed in all adolescents by the same urologist. Varicocele was diagnosed by scrotal palpation, with the adolescent in the standing position and in a controlled temperature environment $\left(23-25^{\circ} \mathrm{C}\right)$, according to the American Society for Reproductive Medicine (ASRM) guidelines (Practice Committee of the American Society for Reproductive Medicine and Society for Male Reproduction and Urology 2014).

Semen samples of all adolescents were collected by masturbation after ejaculatory abstinence of 2-7 days. After semen liquefaction, the sample was divided into two aliquots. The first was used for semen analysis, according to the World Health Organization (WHO) criteria (2010) (World Health Organization, Department of Reproductive Health and Research 2010). Semen volume was assessed with the aid of a serological pipette and after $\mathrm{pH}$ testing strips were used. Motility was assessed manually by Horwell (Arnold R. Horwell Limited ${ }^{\circledR}$, London) chamber, and the sperm were classified as progressive, non-progressive and immotile. Vitality was performed in samples which the progressive motility and non-progressive were less than $40 \%$; semen aliquots were stained with eosin-nigrosin (Sigma-Aldrich) and classified as alive (white spermatozoa) and dead (pink spermatozoa). Sperm concentration was assessed by a modified Neubauer chamber (Herka ${ }^{\oplus}$, Germany), and the result was expressed in millions per milliliter. For morphological evaluations, the kit Panotico (Laborclin ${ }^{\circledast}$, Brazil) was used to stained sperm smears, and the Kruger's criteria was used. Round cells were determined using a modified Neubauer chamber (Herka ${ }^{\circledR}$, Germany) in semen samples diluted in $0.9 \%$ saline, and leukocytes were detected by peroxidase test. All analyses were performed by one experienced technician. The second aliquot was centrifuged at $16,000 \boldsymbol{g}$ for 15 min to separate the seminal plasma from the cellular debris. The seminal plasma was then frozen and stored at $-20^{\circ} \mathrm{C}$ without cryoprotectants until lipid peroxidation and antioxidants activity analyses. All adolescents collected two samples, with a minimum interval of 7 days and maximum of 15 days between collections. Venous blood samples were also collected from all adolescents after the first semen collection and were centrifuged at $300 \boldsymbol{g}$ for $10 \mathrm{~min}$. The serum was then frozen at $-20^{\circ} \mathrm{C}$ until lipid peroxidation analysis.

Inclusion criteria were male adolescents aged between 15 and 17 years, with full sexual maturity (Tanner stage V) who have already initiated the practice of masturbation. Exclusion criteria were: fever reported in the 90 days prior to semen collection; obesity or overweight; smoking, drinking or drug habits; presence or history of systemic diseases (and their treatments); genital malformation; genetic syndrome; testicular dystopia; and history of inguinal-scrotal surgery, orchitis, epididymitis or torsion of the spermatic cord. Adolescents presenting semen volume below $0.5 \mathrm{~mL}$, leukocitospermia and/or azoospermia, or those without varicocele, but with altered semen analysis were also excluded. Due to the applied exclusion criteria, 38 adolescents were excluded from the study.

Based on the presence of varicocele and on semen analysis, the 90 adolescents were further divided into 3 groups: (i) control group, composed by 27 adolescents without varicocele or with varicocele grade I, without any alteration in semen, (ii) varicocele and normal semen (VNS), with 46 adolescents presenting with varicocele grades II and/or III and without alterations in semen, and (iii) varicocele and altered semen (VAS), consisting of 17 adolescents with varicocele grades II and/or III and at least one alteration in semen. Control group included men with varicocele grade I, because our group has previously demonstrated that semen variables and sperm function of adolescents with varicocele grade I do not differ from those of adolescents without varicocele (Mori et al. 2008).

\section{Lipid peroxidation evaluation in seminal plasma}

Semen lipid peroxidation was determined using the method previously described by Ohkawa et al. for the determination of lipid peroxidation products, mainly MDA (Ohkawa et al. 1979). The method is based on the reaction of two molecules of thiobarbituric acid with one molecule of malondialdehyde, at high temperatures and low $\mathrm{pH}$, resulting in a pink chromogen that can be quantified with a spectrophotometer. Briefly, $500 \mu \mathrm{L}$ of seminal plasma were added to $1000 \mu \mathrm{L}$ of $10 \%$ (w:v in water) trichloroacetic acid (TCA) and centrifuged at $16,000 \boldsymbol{g}$ for $15 \mathrm{~min}$ at $15^{\circ} \mathrm{C}$ for protein removal. After, $500 \mu \mathrm{L}$ of the supernatant was added to $500 \mu \mathrm{L}$ of $1 \%$ (v:v in $0.05 \mathrm{M}$ sodium hydroxide) TBA in another tube, which was maintained for $10 \mathrm{~min}$ in boiling water $\left(90-100^{\circ} \mathrm{C}\right)$. Thereafter, the samples were cooled on ice to stop the chemical reaction. The TBARS molecules were quantified by a spectrophotometer (Ultrospec 3300pro, Healthcare ${ }^{\circledast} \mathrm{GE}$ ) at a wavelength of 532 $\mathrm{nm}$. Results were compared to a standard curve previously prepared with a standard solution of malondiadehyde. The TBARS concentration was determined using the value of $1.56 \times 10^{5} / \mathrm{M} \mathrm{cm}$ as the MDA extinction coefficient. Lipid peroxidation in the seminal plasma was described as TBARS in nanograms/milliliter of seminal plasma. The TBARS 
values were also normalized by the sperm concentration, because the sperm is the major source of MDA production in semen. Therefore, the TBARS $(\mathrm{ng} / \mathrm{mL})$ was divided by the concentration of sperm $\left(10^{6} / \mathrm{mL}\right)$ to obtain the TBARS $/ 10^{6}$ sperm values (Lacerda et al. 2011, Sposito et al. 2017).

\section{Evaluation of lipid peroxidation in serum}

Lipid peroxidation levels in serum were determined using a modified protocol from Ohkawa et al (Ohkawa et al. 1979). Thus, $200 \mu \mathrm{L}$ of serum were added to $400 \mu \mathrm{L}$ of a solution containing: $10 \%(\mathrm{v}: \mathrm{v})$ TFA , $0.375 \%(\mathrm{v}: \mathrm{v}) \mathrm{TBA}$, and $0.25 \mathrm{~N}$ hydrochloric acid $(\mathrm{HCl})$.

After homogenization, the samples were placed in a water bath (Scientific ${ }^{\oplus}$ Fisher, Isotemp 205) at $100^{\circ} \mathrm{C}$ for $15 \mathrm{~min}$. Then, the samples were cooled in an ice bath at $0^{\circ} \mathrm{C}$ for $5 \mathrm{~min}$, and $500 \mu \mathrm{L}$ of $n$-butanol (Synth ${ }^{\circledast}$, A1077.01) were added. After homogenization in vortex, the samples were centrifuged (Eppendorf 5804R) for $15 \mathrm{~min}$ at $2500 \mathrm{~g}$. The supernatant was collected and transferred in duplicate to a 96-well microplate. TBARS molecules were then quantified by spectrophotometry (ELx800 Absorbance Microplate Reader, Biotek, Vermont, USA) at a wavelength of $532 \mathrm{~nm}$. Results were compared to a standard curve previously prepared with a standard solution of malondiadehyde. Lipid peroxidation levels were described in TBARS nanogram per milliliter of serum.

\section{Antioxidant enzymes activity in seminal plasma}

\section{Catalase activity}

Catalase activity was assessed indirectly by hydrogen peroxide $\left(\mathrm{H}_{2} \mathrm{O}_{2}\right)$ consumption (Nichi et al. 2006). In a quartz cuvette (Biocell, California, USA), $5 \mu \mathrm{L}$ of seminal plasma were mixed to $20 \mu \mathrm{L}$ of milli-Q water (Millipore Corporation), $25 \mu \mathrm{L}$ of TE buffer ( $50 \mathrm{mM}$ trisaminomethane and $25 \mathrm{mM}$ EDTA, pH 8.0) and $450 \mu \mathrm{L}$ of $900 \mu \mathrm{M}$ hydrogen peroxide. Samples were then maintained at $30^{\circ} \mathrm{C}$ for $5 \mathrm{~min}$, and the absorbance was measured every $5 \mathrm{~s}$, and the curve of $\mathrm{H}_{2} \mathrm{O}_{2}$ consumption was compared to a blank. Samples were read in a spectrophotometer (Ultrospec 3300pro, Healthcare ${ }^{\circledast}$ GE) and calculations considered $0.071 / \mathrm{M} \mathrm{cm}$ as the molar extinction coefficient of hydrogen peroxide. Catalase activity was calculated based on the formula $\mathrm{CAT}=($ initial absorbance - final $) / 0.071 \times$ dilution. Results were expressed as $\mathrm{UI} / \mathrm{mL}$.

\section{Glutathione peroxidase (GPx) activity}

GPx enzyme activity was assessed indirectly by measuring the NAD phosphate (NADPH) consumption, using the Beutler protocol modified by Bittencourt (de Bittencourt et al. 1998). This method is based on measuring the consumption of NADPH; the reaction between a hydroperoxide and reduced glutathione (GSH) that is catalyzed by the GSPH-Px together with the enzyme glutathione reductase (GSSGr) is induced. This reaction causes the conversion of glutathione disulfide (GSSH glutathione oxidized) to GSH, which in turn consumes NADPH (measured with a spectrophotometer). Briefly, $100 \mu \mathrm{L}$ of seminal plasma were mixed to $20 \mu \mathrm{L}$ of 0.25 $\mathrm{mM}$ sodium azide, $20 \mathrm{~mL}$ of $0.25 \mathrm{UI}$ GSSGr, $100 \mathrm{~mL}$ of $0.12 \mathrm{mM} \mathrm{GSH}, 660 \mu \mathrm{L}$ of a solution containing $143 \mathrm{mM}$ sodium phosphate and $6.3 \mathrm{mM}$ EDTA, and $1000 \mu \mathrm{L}$ of 0.12 mM NADPH. Then, the cuvette was stirred in vortex and maintained in the spectrophotometer (Ultrospec 3300pro, Healthcare ${ }^{\circledast} \mathrm{GE}$ ) for 300 s to stabilize the reaction. After, 100 $\mathrm{mL}$ of tert-butyl hydroperoxide in $1.2 \mathrm{mM}$ hydroperoxide (TBHP) were added. The consumption curve of NADPH was measured in a wavelength of $340 \mathrm{~nm}$ for $10 \mathrm{~min}$ at $37^{\circ} \mathrm{C}$ (measured at every $5 \mathrm{~s}$ ) and compared to a blank. Calculations considered the value of $0.0062 / \mathrm{M} \mathrm{cm}$ as the molar extinction coefficient of NADPH. Glutathione peroxidase activity was calculated based on the formula: GPxactivity $=($ final absorbance - initial $) / 0.0062 \times$ dilution . Results were expressed as UI/mL.

\section{Superoxide dismutase (SOD) activity}

Superoxide dismutase was indirectly assessed by the rate of cytochrome c reduction by superoxide anion $\left(\mathrm{O}_{2}-\right)$ (Flohé \& Otting, 1984). The xanthine-xanthine oxidase system continuously generated $\mathrm{O}^{2-}$ (that reduced cytochrome C). The SOD present in the sample competed with the cytochrome $\mathrm{C}$ by converting the superoxide free radical to $\mathrm{H}_{2} \mathrm{O}_{2}$ and $\mathrm{O}^{2-}$, thereby slowing the rate of cytochrome $\mathrm{C}$ reduction. The reaction medium was composed by $1 \mathrm{mM}$ cytochrome c, $50 \mathrm{mM}$ xanthine, $100 \mathrm{mM}$ EDTA and $50 \mu \mathrm{M}$ sodium phosphate buffer ( $\mathrm{pH}$ 7.8). The adjustment by the required activity of xanthine oxidase (Sigma-Aldrich ${ }^{\circledast}$ ) for the generation of $\mathrm{O}_{2}$ - and for the reduction of cytochrome c was performed as 0.025 absorbance units per minute, because 1 unit of total SOD activity corresponds to $50 \%$ of this value. The assay was performed at $550 \mathrm{~nm}$ and $25^{\circ} \mathrm{C}$ for $5 \mathrm{~min}$. Therefore, SOD activity in the sample decreased the rate of cytochrome reduction when compared to the blank. To calculate SOD activity, we used [ $\Delta$ absorbance

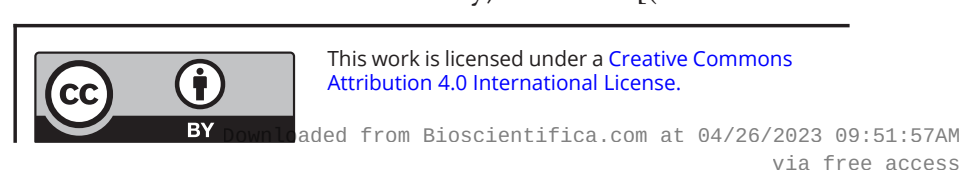


of sample -0.025$) / 0.0125) \times$ dilution]. The results were expressed as $\mathrm{U} / \mathrm{mL}$.

\section{Statistical analysis}

Data analysis was performed using the SPSS 18.0 for Windows ${ }^{\circledR}$ (SPSS). Initially, normality of data distribution was evaluated by Kolmogorov-Smirnov test. Non-normally distributed data were transformed to their logarithmic values.

Groups were compared by ANOVA followed by post hoc Tukey HSD. Transformed data that still did not obey the normality assumption were compared using the KruskalWallis test, followed by post hoc Tamhane. For these variables, data were presented as median and interquartile range. For correlation analysis, we used the Pearson test (variables that did not show normality were previously transformed). For the entire study, an $\alpha$ of $5 \%$ was adopted. Effect size and power of the study were calculated using G*Power 3.1.9.4.

\section{Results}

\section{Study groups and excluded samples}

We recruited 128 adolescents, of which 38 were excluded due to the exclusion criteria (samples that would add bias to our study): 8 with inguinal herniorrhaphy, 8 with semen alteration without varicocele, 7 with leukocytospermia, 3 with semen volume less than $0.5 \mathrm{~mL}, 2$ with obesity, 2 with testicular asymmetry, 1 with overweight, 1 with low testicular volume, 1 with cryptorchidism, 1 with hydrocele, 2 with azoospermia, 1 with orchiepididymitis and 1 with testicular asymmetry+ inguinal herniorrhaphy+ leukocytospermia. Therefore, groups were formed as 27 adolescents in control, 46 in varicocele normal semen and 17 varicocele altered semen. Despite the number of patients be 27,46 and 17, respectively, when we calculate the effect size and the power of the study, we observe that the effect size is intermediate (Cohen's $d=0.66$ ). Therefore, there is a high magnitude of the real difference between groups. Furthermore, the power observed is $80 \%$. Thus, there is a high chance of the sample to detect a difference that we hope to observe in the population.

\section{Seminal quality and clinical characteristics}

No differences between the groups were observed regarding age $(P=0.984)$, right and left testicular volumes
$(P=0.363$ and $P=0.168)$, ejaculatory abstinence length $(P=0.901)$, semen volume $(P=0.727)$, and concentration of round cells and neutrophils $(P=0.139$ and $P=0.181)$. However, the VAS group presented a decrease in sperm concentration $(P<0.001)$, progressive motility $(P=0.020)$ and morphology $(P<0.001)$, as well as decreased total sperm count $(P<0.001)$, motile sperm concentration $(P<0.001)$ and total count of morphologically normal motile sperm $(P<0.001)$ compared to control and VNS (Table 1).

\section{Antioxidants enzyme activity and lipid peroxidation levels}

The catalase activity was undetectable in seminal plasma by the employed method. Seminal enzymatic activities of superoxide dismutase (SOD) and glutathione peroxidase (GPx) did not differ between groups $(P=0.914$ and $P=0.497)$ (Table 2). The VAS group presented an increase of TBARS $/ 10^{6}$ sperm compared to control and VNS $(P<0.001)$ and TBARS in serum did not differ between groups $(P=0.459)$.

\section{Correlations}

The correlation index between clinical and seminal variables, SOD and GPx activities and lipid peroxidation levels in semen (TBARS $/ 10^{6}$ sperm) and serum $(\mathrm{ng} / \mathrm{mL})$ in the control, VNS and VAS groups are demonstrated in the Table 3. Sperm concentration was negatively correlated to TBARS $/ 10^{6}$ sptz in all groups: control $(\mathrm{r}=-0.765, P<0.001)$, VSN $(r=-0.680, P<0.001)$ and VSA $(r=-0.503, P=0.047)$. Also, Log TMNM was negatively correlated to TBARS $/ 10^{6}$ sptz in the control group $(\mathrm{r}=-0.835, P<0.001)$, in the VNS group $(\mathrm{r}=-0.791, P<0.001)$ and in VAS group $(\mathrm{r}=-0.769$, $P<0.001)$. Non-progressive motility (NP) was negatively correlated to GPx activity $(\mathrm{r}=-0.421, P=0.029)$ only in the control group. On the other hand, non-progressive motility (NP) was positively correlated to TBARS $/ 10^{6} \mathrm{sptz}$ $(\mathrm{r}=0.530, P=0.035)$ only in the VAS group. TBARS $/ 10^{6} \mathrm{sptz}$ was negatively correlated to morphology in the control $\operatorname{group}(\mathrm{r}=-0.516, P=0.007)$ and VNS $(\mathrm{r}=-0.451, P=0.002)$. Finally, ejaculate volume was negatively correlated to GPx activity $(\mathrm{r}=-0.580, P=0.015)$ only in the VAS group.

\section{Discussion}

Varicocele, due to several and interrelated mechanisms, may cause testicular dysfunction, compromising the process of spermatogenesis and leading to decreased 


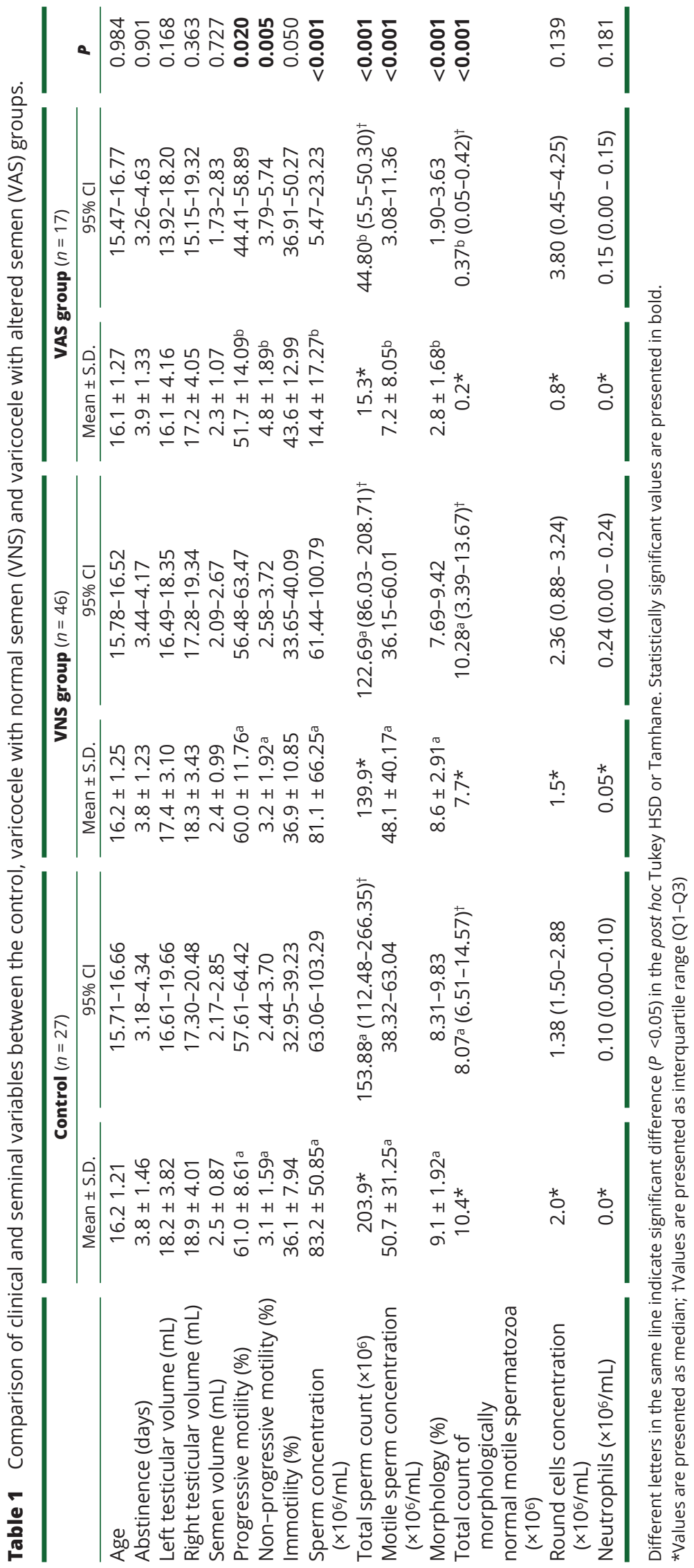



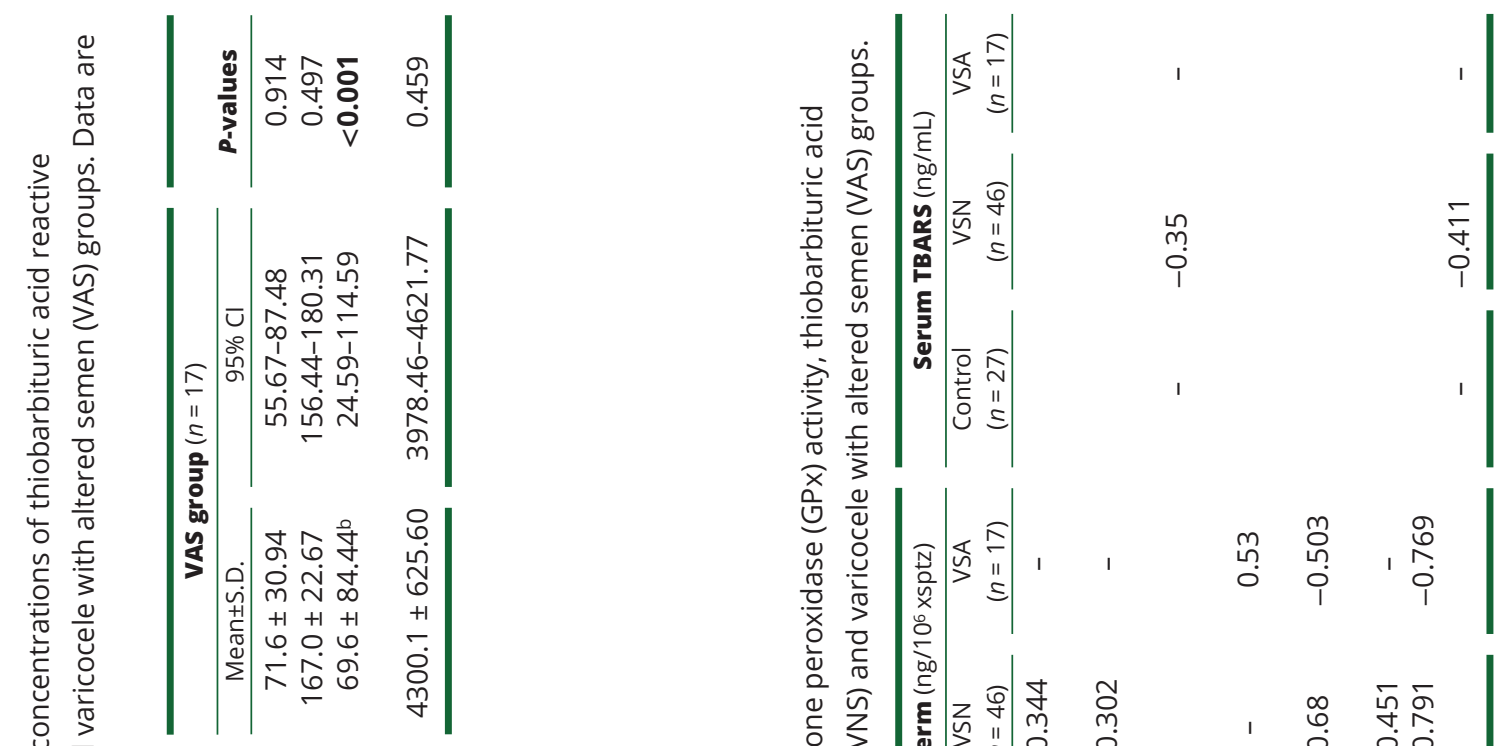

중

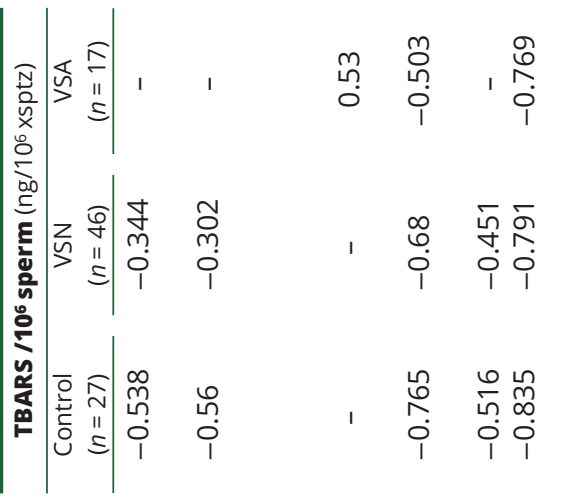

崩

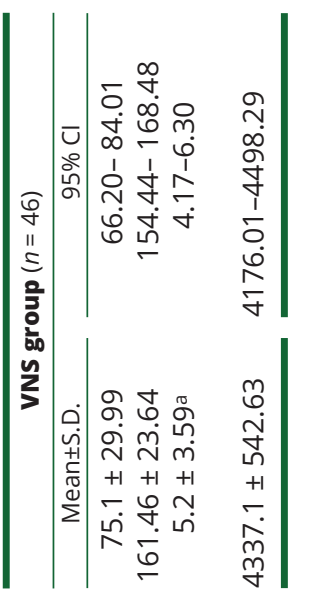

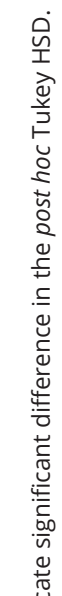

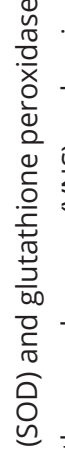

$\stackrel{n}{u}$

崩

岳

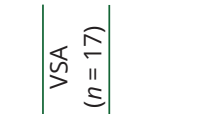

$\infty$
$\stackrel{n}{0}$
$\substack{1 \\ 1}$

d)

믓

일 등

放

망

ดิ

证

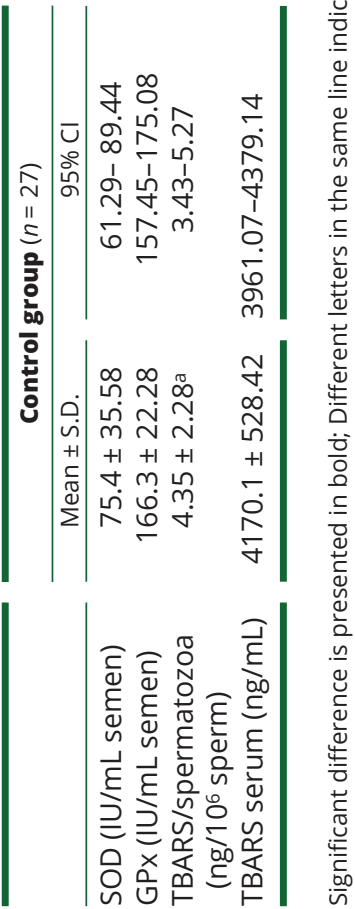

$\frac{\text { d }}{\frac{0}{0}}$

$\frac{\sqrt{2}}{>}$

$\frac{\pi}{2}$

है

ơ

है

氖

든

बิ

$\sum_{0}^{\infty} \frac{E}{d}$

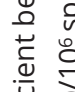

旁 䨌

党
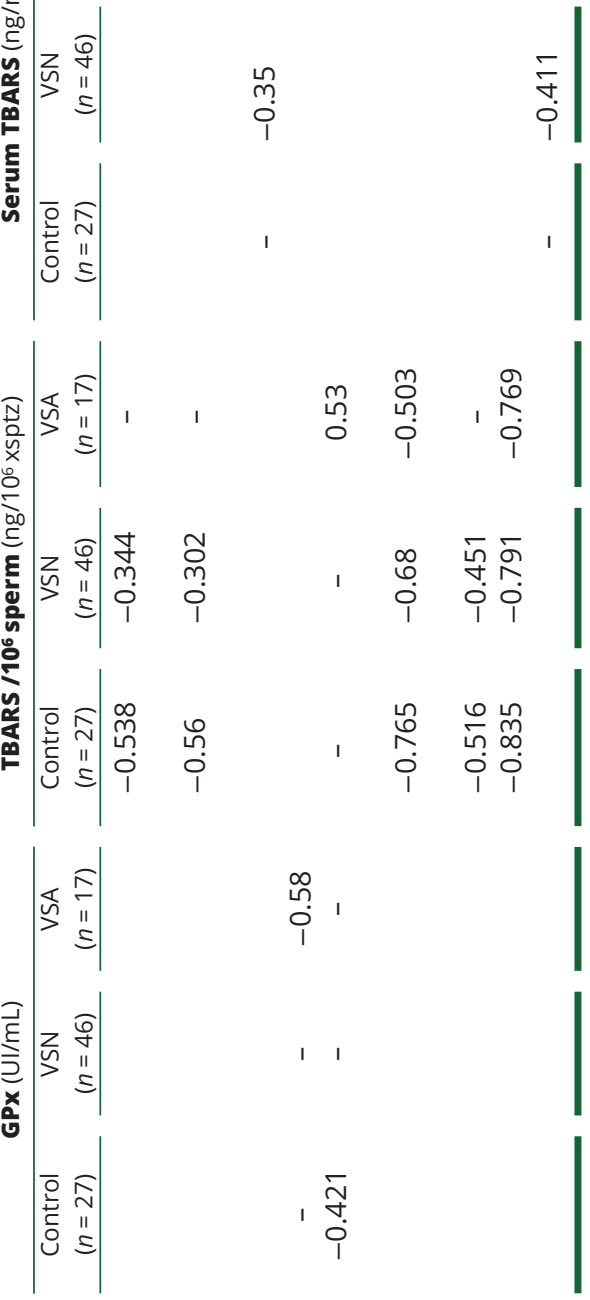

은 는

竞

仓ั

m

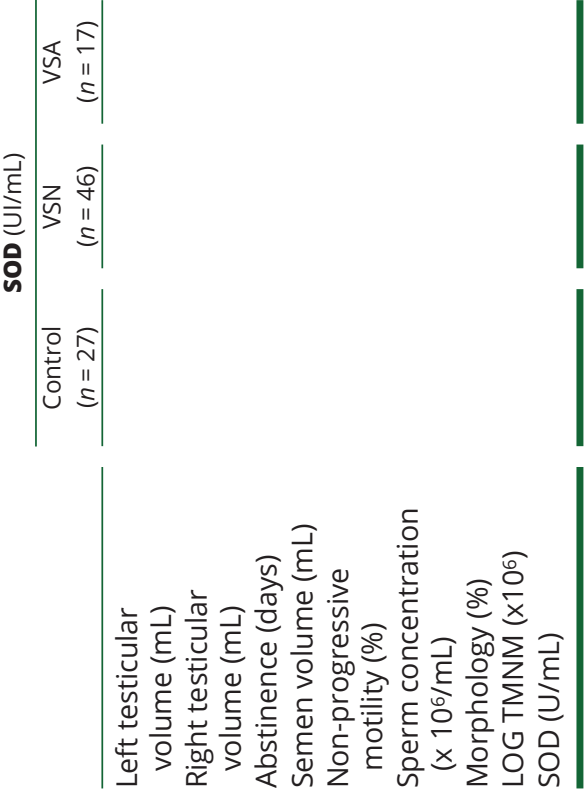


semen quality and/or sperm function, thus impairing male fertility (Hassanin et al. 2018). However, as not every adult with varicocele is infertile, the study of the mechanisms associated with the onset of varicocele-related infertility may increase the understanding of the early mechanisms of testicular injury.

Several mechanisms have been proposed to be involved in the pathophysiology of varicocele, which we can highlight the oxidative stress (Agarwal et al. 2009, Robinson et al. 2010). Oxidative stress is described as the imbalance between the production of ROS and the antioxidant protection of semen, in favor of the oxidant (Aitken \& Krausz 2001). The production of ROS by sperm is a normal physiological process, being important for hyperactivation regulation, acrosome reaction and the fusion between sperm and oocyte. Despite the physiologically normal effect of ROS on the sperm cell, an imbalance between the production and the elimination of these species in the semen causes harmful effects to the sperm. The semen's antioxidant mechanisms, whether enzymatic or nonenzymatic, are responsible for trying to maintain this oxidative balance and avoid such damage (de Lamirande \& Gagnon 1993, Aitken et al. 1997, de Lamirande et al. 1997).

For a better understanding of the role of oxidative stress in the establishment of testicular dysfunction in varicocele, the present study aimed to evaluate if there is an association between semen lipid peroxidation levels and semen quality in adolescents with and without varicocele and the role of seminal enzymatic antioxidants in varicocele-induced oxidative stress. In our study, increased lipid peroxidation levels were observed in the varicocele altered semen (VAS) group compared to the other groups. However, no differences were observed between the groups regarding superoxide dismutase and glutathione peroxidase activities in the seminal plasma and TBARS levels in the serum of adolescents.

It is possible to suggest that the seminal oxidative stress observed in the VAS group can result from the testicular venous stasis caused by varicocele, leading to heat stress and accumulation of toxic metabolites, causing an increase in ROS production (Agarwal et al. 2012, Hamada et al. 2013, Ritchie \& Ko 2021). Moreover, it is important to highlight that the substrate for the seminal lipid peroxidation in this study was exclusively the sperm membrane, given that samples with leukocytospermia were excluded. Therefore, because in the VAS group the sperm concentration is decreased, it is suggested that in this group each sperm is being subjected to lipid peroxidation more intensely than in the other groups.
Although seminal lipid peroxidation levels were increased, no differences were observed between the groups regarding TBARS levels in serum. Furthermore, the enzymatic activities of SOD and GPx in the seminal plasma did not differ between the groups, although the values of TBARS $/ 10^{6}$ sptz were much greater in the VAS group. Thus, this increased oxidative stress in the VAS group does not seem to be related to a decreased seminal plasma antioxidant capacity, as we had hypothesized, nor to a systemic oxidative stress in adolescents with varicocele. Although some studies have demonstrated a decreased semen total antioxidant capacity (TAC), and SOD, GPx and catalase levels in seminal plasma of men with varicocele (Pasqualotto et al. 2008, Mostafa et al. 2012), and an increase in the concentration of seminal antioxidants after varicocelectomy (Chen et al. 2008), these studies were performed in adult men, not in adolescents. Therefore, we can speculate that the reduction in antioxidant enzyme activity caused by varicocele, can be a process that takes place over time (Andriollo-Sanchez et al. 2005, NguyenPowanda \& Robaire 2020).

In the present study, we observed a negative correlation between TBARS $/ 10^{6}$ sptz and sperm concentration in all groups; also, a negative correlation between TBARS $/ 10^{6} \mathrm{sptz}$ and morphology was observed in control and VNS groups, but this correlation was lost in the VAS group. This can be explained by a dysregulation in the apoptosis process in the testis of men with varicocele (Fujisawa et al. 1999), which may have started in adolescence, as evidenced in a study that showed the presence of proteins with important role in DNA repair and promoting apoptosis (SMG1 and IBP3) exclusively in adolescents with varicocele and altered semen (Zylbersztejn et al. 2013).

There was also a positive correlation between nonprogressive motility and TBARS $/ 10^{6}$ sptz only in the VAS group, which again indicates that this group has a greater damage to the sperm membrane when compared to control groups and VNS. Although it is well established that high concentrations of ROS decrease sperm motility (Alahmar 2019), the exact mechanisms through which this occurs are not completely understood. One hypothesis suggests that $\mathrm{H}_{2} \mathrm{O}_{2}$ diffuses through the cell membrane and inhibits the activity of some vital enzymes such as glucose6-phosphate dehydrogenase (Gomez et al. 1996).

The correlation of oxidative stress with sperm concentration, motility, and especially morphology corroborates the finding of a negative correlation between Log TMMN and TBARS $/ 10^{6}$ sptz in the control group, the VNS group and VAS group. This shows that when these

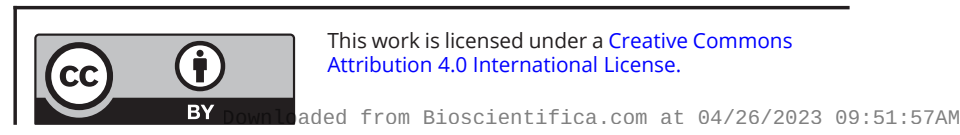


three variables are analyzed together, the correlation with oxidative stress is even stronger.

Shalini and Bansal considered the GPx an oxidative stress marker since its activity seems to be altered in response to a stress (Shalini \& Bansal 2005). Accordingly, in the present study, a negative correlation was observed between non-progressive motility (NP) and GPx activity only in the control group, indicating that this GPx response to stress may be limited. The fact that this correlation was lost in the groups with varicocele, and taking into account that the activity of antioxidant enzymes did not differ between the groups, suggests that the presence of the disease can result in a higher testicular stress than the physiological enzymatic responsiveness. This correlation only in the control group could also indicate that the system is in oxidative homeostasis: the GPx would be produced in response to any stress and would be effective in keeping the ideal patterns of motility. Finally, new controlled and carefully designed studies are needed to clarify how oxidative stress and its markers are associated with decreased fertility potential and thereby to identify which adolescents would benefit from early correction of varicocele.

\section{Conclusion}

In conclusion, varicocele leads to seminal oxidative stress that is not caused by decreased activity of antioxidant enzymes. Bearing this in mind, it is important to appropriately address varicocele in order to remove prooxidative conditions, rather than to focus on re-establishing antioxidant activity.

\section{Declaration of interest}

The authors declare that there is no conflict of interest that could be perceived as prejudicing the impartiality of the research reported.

\section{Funding}

This research did not receive any specific grant from any funding agency in the public, commercial or not-for-profit sector.

\section{Author contribution statement}

V B; D M S Conception, designing, conducting and writing the manuscript. V B; M N Sampling and sperm analyses. M P A; P I Data interpretation and revision of the article. $\mathrm{R} P \mathrm{~B}$ Data interpretation and statistical analysis. All authors read and approved the final manuscript.

\section{Acknowledgement}

The work was done at Universidade Federal de São Paulo (UNIFESP) - Sao Paulo Hospital, Sao Paulo, Brazil.

\section{References}

Agarwal A \& Majzoub A 2017 Laboratory tests for oxidative stress. Indian Journal of Urology 33 199-206. (https://doi.org/10.4103/iju.IJU_9_17)

Agarwal A, Sharma RK, Desai NR, Prabakaran S, Tavares A \& Sabanegh E 2009 Role of oxidative stress in pathogenesis of varicocele and infertility. Urology 73 461-469. (https://doi. org/10.1016/j.urology.2008.07.053)

Agarwal A, Hamada A \& Esteves SC 2012 Insight into oxidative stress in varicocele-associated male infertility: Part 1. Nature Reviews: Urology 9 678-690. (https://doi.org/10.1038/nrurol.2012.197)

Aitken RJ 2020 Impact of oxidative stress on male and female germ cells; implications for fertility. Reproduction 159 R189-R201. (https://doi. org/10.1530/REP-19-0452)

Aitken RJ \& Krausz C 2001 Oxidative stress, DNA damage and the Y chromosome. Reproduction 122 497-506. (https://doi.org/10.1530/ rep.0.1220497)

Aitken RJ, Fisher HM, Fulton N, Gomez E, Knox W, Lewis B \& Irvine S 1997 Reactive oxygen species generation by human spermatozoa is induced by exogenous NADPH and inhibited by the flavoprotein inhibitors diphenylene iodonium and quinacrine. Molecular Reproduction and Development 47 468-482. (https://doi.org/10.1002/(SICI)1098-2795(199708)47:4<468::AIDMRD14>3.0.CO;2-S)

Aitken RJ, Whiting S, De Iuliis GN, McClymont S, Mitchell LA \& Baker MA 2012 Electrophilic aldehydes generated by sperm metabolism activate mitochondrial reactive oxygen species generation and apoptosis by targeting succinate dehydrogenase. Journal of Biological Chemistry 287 33048-33060. (https://doi.org/10.1074/jbc. M112.366690)

Alahmar AT 2019 Role of oxidative stress in male infertility: an updated review. Journal of Human Reproductive Sciences 12 4-18. (https://doi. org/10.4103/jhrs.JHRS_150_18)

Andriollo-Sanchez M, Hininger-Favier I, Meunier N, Venneria E, O'Connor JM, Maiani G, Coudray C \& Roussel AM 2005 Agerelated oxidative stress and antioxidant parameters in middle-aged and older European subjects: the ZENITH study. European Journal of Clinical Nutrition 59(Supplement 2) S58-S62. (https://doi.org/10.1038/ sj.ejcn.1602300)

Benedetti S, Tagliamonte MC, Catalani S, Primiterra M, Canestrari F, De Stefani S, Palini S \& Bulletti C 2012 Differences in blood and semen oxidative status in fertile and infertile men, and their relationship with sperm quality. Reproductive Biomedicine Online 25 300-306. (https://doi.org/10.1016/j.rbmo.2012.05.011)

Brugh VM \& Lipshultz LI 2004 Male factor infertility: evaluation and management. Medical Clinics of North America 88 367-385. (https:// doi.org/10.1016/S0025-7125(03)00150-0)

Brugh VM, Matschke HM \& Lipshultz LI 2003 Male factor infertility. Endocrinology and Metabolism Clinics of North America 32 689-707. (https://doi.org/10.1016/s0889-8529(03)00047-1)

Chen SS, Huang WJ, Chang LS \& Wei YH 2008 Attenuation of oxidative stress after varicocelectomy in subfertile patients with varicocele. Journal of Urology 179 639-642. (https://doi.org/10.1016/j. juro.2007.09.039)

Cho CL, Esteves SC \& Agarwal A 2016 Novel insights into the pathophysiology of varicocele and its association with reactive oxygen species and sperm DNA fragmentation. Asian Journal of Andrology 18 186-193. (https://doi.org/10.4103/1008-682X.170441)

Clavijo RI, Carrasquillo R \& Ramasamy R 2017 Varicoceles: prevalence and pathogenesis in adult men. Fertility and Sterility $\mathbf{1 0 8}$ 364-369. (https://doi.org/10.1016/j.fertnstert.2017.06.036)

de Bittencourt PI, Miyasaka CK, Curi R \& Williams JF 1998 Effects of the antiproliferative cyclopentenone prostaglandin A1 on glutathione metabolism in human cancer cells in culture. Biochemistry and Molecular Biology International 45 1255-1264. (https://doi. org/10.1080/15216549800203472) 
de Lamirande E \& Gagnon C 1993 Human sperm hyperactivation in whole semen and its association with low superoxide scavenging capacity in seminal plasma. Fertility and Sterility 59 1291-1295. (https://doi.org/10.1016/S0015-0282(16)55992-5)

de Lamirande E, Jiang H, Zini A, Kodama H \& Gagnon C 1997 Reactive oxygen species and sperm physiology. Reviews of Reproduction 2 48-54. (https://doi.org/10.1530/ror.0.0020048)

Flohé L \& Otting F 1984 Superoxide dismutase assays. Methods in Enzymology 105 93-104. (https://doi.org/10.1016/s00766879(84)05013-8)

Fujisawa M, Hiramine C, Tanaka H, Okada H, Arakawa S \& Kamidono S 1999 Decrease in apoptosis of germ cells in the testes of infertile men with varicocele. World Journal of Urology 17 296-300. (https://doi.org/10.1007/s003450050149)

Gomez E, Buckingham DW, Brindle J, Lanzafame F, Irvine DS \& Aitken RJ 1996 Development of an image analysis system to monitor the retention of residual cytoplasm by human spermatozoa: correlation with biochemical markers of the cytoplasmic space, oxidative stress, and sperm function. Journal of Andrology 17 276-287.

Gorelick JI \& Goldstein M 1993 Loss of fertility in men with varicocele. Fertility and Sterility 59 613-616. (https://doi.org/10.1016/S00150282(16)55809-9)

Hamada A, Esteves SC \& Agarwal A 2013 Insight into oxidative stress in varicocele-associated male infertility: Part 2. Nature Reviews: Urology 10 26-37. (https://doi.org/10.1038/nrurol.2012.198)

Hassanin AM, Ahmed HH \& Kaddah AN 2018 A global view of the pathophysiology of varicocele. Andrology 6 654-661. (https://doi. org/10.1111/andr.12511)

Jensen CFS, Østergren P, Dupree JM, OhI DA, Sønksen J \& Fode M 2017 Varicocele and male infertility. Nature Reviews: Urology 14 523-533. (https://doi.org/10.1038/nrurol.2017.98)

Lacerda JI, Del Giudice PT, da Silva BF, Nichi M, Fariello RM, Fraietta R, Restelli AE, Blumer CG, Bertolla RP \& Cedenho AP 2011 Adolescent varicocele: improved sperm function after varicocelectomy. Fertility and Sterility 95 994-999. (https://doi. org/10.1016/j.fertnstert.2010.10.031)

Mori MM, Bertolla RP, Fraietta R, Ortiz V \& Cedenho AP 2008 Does varicocele grade determine extent of alteration to spermatogenesis in adolescents? Fertility and Sterility 90 1769-1773. (https://doi. org/10.1016/j.fertnstert.2007.09.052)

Mostafa T, Anis T, El Nashar A, Imam H \& Osman I 2012 Seminal plasma reactive oxygen species-antioxidants relationship with varicocele grade. Andrologia 44 66-69. (https://doi.org/10.1111/j.1439. 0272.2010.01111.x)

Nguyen-Powanda P \& Robaire B 2020 Oxidative stress and reproductive function in the aging male. Biology 9 282-296. (https:// doi.org/10.3390/biology9090282)

Nichi M, Bols PEJ, Züge RM, Barnabe VH, Goovaerts IGF, Barnabe RC \& Cortada CNM 2006 Seasonal variation in semen quality in Bos indicus and Bos taurus bulls raised under tropical conditions. Theriogenology 66 822-828. (https://doi.org/10.1016/j. theriogenology.2006.01.056)

Ohkawa H, Ohishi N \& Yagi K 1979 Assay for lipid peroxides in animal tissues by thiobarbituric acid reaction. Analytical Biochemistry 95 351-358. (https://doi.org/10.1016/0003-2697(79)90738-3)
Pasqualotto FF, Sundaram A, Sharma RK, Borges E Pasqualotto EB \& Agarwal A 2008 Semen quality and oxidative stress scores in fertile and infertile patients with varicocele. Fertility and Sterility 89 602-607. (https://doi.org/10.1016/j.fertnstert.2007.03.057)

Practice Committee of the American Society for Reproductive Medicine, Society for Male Reproduction and Urology \& Society for Male Reproduction and Urology 2014 Report on varicocele and infertility: a committee opinion. Fertility and Sterility 102 1556-1560. (https://doi. org/10.1016/j.fertnstert.2014.10.007)

Ritchie C \& Ko EY 2021 Oxidative stress in the pathophysiology of male infertility. Andrologia 53 e13581. (https://doi.org/10.1111/and.13581)

Robinson SP, Hampton LJ \& Koo HP 2010 Treatment strategy for the adolescent varicocele. Urologic Clinics of North America 37 269-278. (https://doi.org/10.1016/j.ucl.2010.03.011)

Romeo C, Ientile R, Impellizzeri P, Turiaco N, Teletta M, Antonuccio P, Basile M \& Gentile C 2003 Preliminary report on nitric oxide-mediated oxidative damage in adolescent varicocele. Human Reproduction 18 26-29. (https://doi.org/10.1093/humrep/deg004)

Shalini S \& Bansal MP 2005 Role of selenium in regulation of spermatogenesis: involvement of activator protein 1. Biofactors 23 151-162. (https://doi.org/10.1002/biof.5520230304)

Sposito C, Camargo M, Tibaldi DS, Barradas V, Cedenho AP, Nichi M, Bertolla RP \& Spaine DM 2017 Antioxidant enzyme profile and lipid peroxidation products in semen samples of testicular germ cell tumor patients submitted to orchiectomy. International Braz $j$ Urol 43 644-651. (https://doi.org/10.1590/S1677-5538.IBJU.2016.0323)

Szczykutowicz J, Kałuża A, Kaźmierowska-Niemczuk M \& FerensSieczkowska M 2019 The potential role of seminal plasma in the fertilization outcomes. BioMed Research International 20195397804. (https://doi.org/10.1155/2019/5397804)

Tsikas D 2017 Assessment of lipid peroxidation by measuring malondialdehyde (MDA) and relatives in biological samples: analytica and biological challenges. Analytical Biochemistry 524 13-30. (https:// doi.org/10.1016/j.ab.2016.10.021)

Türkyilmaz Z, Gülen S, Sönmez K, Karabulut R, Dinçer S, Can Başaklar A \& Kale N 2004 Increased nitric oxide is accompanied by lipid oxidation in adolescent varicocele. International Journal of Andrology 27 183-187. (https://doi.org/10.1111/j.13652605.2004.00474.x)

Witt MA \& Lipshultz LI 1993 Varicocele: a progressive or static lesion? Urology 42 541-543. (https://doi.org/10.1016/0090-4295(93)90268-f)

World Health Organization, Department of Reproductive Health and Research 2010 WHO Laboratory Manual for the Examination and Processing of Human Semen.

Zylbersztejn DS, Andreoni C, Del Giudice PT, Spaine DM, Borsari L, Souza GHMF, Bertolla RP \& Fraietta R 2013 Proteomic analysis of seminal plasma in adolescents with and without varicocele. Fertility and Sterility 99 92-98. (https://doi.org/10.1016/j. fertnstert.2012.08.048)

Received in final form 16 April 2021

Accepted 6 May 2021

Accepted Manuscript published online 6 May 2021 https://raf.bioscientifica.com

https://doi.org/10.1530/RAF-20-0048

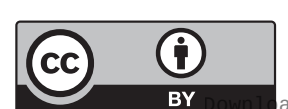

This work is licensed under a Creative Commons Attribution 4.0 International License. 